\title{
Reducing uncertainty and obtaining superior performance by segmentation based on algebraic inequalities
}

\author{
Michael Todinov \\ School of Engineering, Computing and Mathematics \\ Oxford Brookes University, Oxford, UK \\ mtodinov@brookes.ac.uk
}

\begin{abstract}
The paper demonstrates for the first time uncertainty reduction and superior performance through segmentation based on algebraic inequalities. Meaningful interpretation of algebraic inequalities has been used for generating new knowledge in unrelated application domains. Thus, the method of segmentation through an abstract inequality led to a new fundamental theorem related to electrical circuits. The power output from a source with particular voltage, on elements connected in series, is smaller than the total power output from the segmented sources applied to the individual elements. Segmentation attained through the same abstract inequality led to another fundamental theorem related to electrical capacitors. The energy stored by a charge of given size on a single capacitor is smaller than the total energy stored in multiple capacitors with the same equivalent capacity, by segmenting the initial charge over the separate capacitors.

Finally, inequalities based on sub-additive and super-additive functions have been introduced for reducing uncertainty and obtaining superior performance by a segmentation or aggregation of controlling factors. By a meaningful interpretation of sub-additive and superadditive inequalities, superior performance and important properties have been established for processes described by a power-law dependence.
\end{abstract}

Keywords: segmentation; aggregation; algebraic inequalities; uncertainty reduction 


\section{Introduction}

The primary focus of risk research has been on risk assessment and risk prediction rather than on risk reduction. Similarly, methods for uncertainty quantification have been developed rather than methods for reducing uncertainty. It needs to be pointed out that even the available methods for measuring and assessing reliability and risk cannot always be fully implemented in the design for the obvious reason that for new products and processes reliability data are simply unavailable. In the rare cases where reliability data for the components and parts building the systems are available, they are relevant for a particular environment and duty cycle and their mechanical application to another environment and duty cycle, as experience has shown, is of highly questionable value. The lack of predictive capability of the existing reliability tools caused many engineers to lose faith in the tools and discard them as not adding real value to their work.

In standard textbooks on mechanical engineering and design of machine components (Collins, 2003; Norton, 2006; Pahl et al.2007; Childs, 2014; Budynas and Nisbett, 2015; Mott et al, 2018; Gullo and Dixon, 2018; French, 1999; Samuel and Weir, 1999; Thompson, 1999), for example, virtually no discussion exists related to generic methods for reliability improvement and risk and uncertainty reduction.

Why is the engineering design so slow in exploiting the achievements of the reliability and risk science to improve reliability and reduce risk? This is certainly not due to the complexity of the reliability improvement and risk reduction methods. In this respect, the contrast with the more complex generic mathematical methods for stress analysis, kinematic and dynamic analysis of solid bodies and fluids is striking. These mathematical modelling methods are penetrating all aspects of the engineering design. 
The key problem is that the current approach to reliability improvement and risk reduction almost solely relies on knowledge from a specific domain and is conducted exclusively by experts in that specific domain. However, unfamiliarity with domain-independent methods causes many valuable opportunities for reliability improvement and risk reduction to remain invisible to experts in the specific domain. In addition, current technology changes so fast that the domain-specific skills in reliability improvement and risk reduction are outdated almost as soon as they are learned.

In contrast, the domain-independent methods in reliability improvement, risk and uncertainty reduction are higher-order methods that permit applying strategically the same methods and principles in new, constantly changing situations and circumstances.

Most of the well-known domain-independent methods for risk reduction are associated with significant investment. To address this problem, a number of domain-independent risk reduction methods have been proposed in (Todinov, 2019a, 2019b) among which was the method of segmentation whose idea is to prevent failure modes and reduce vulnerability to a single failure, by dividing an object or process into smaller parts.

The present paper develops this method further by obtaining superior performance of systems and processes through segmentation and aggregation based on algebraic inequalities.

There is clear lack of discussion in textbooks on reliability engineering (Modares et al, 2017; Dhillon, 2017; Ebeling, 1997; Lewis, 1996; O'Connor, 2002), related to reducing risk and uncertainty by using algebraic inequalities. This is a surprising omission considering the power of algebraic inequalities in reducing risk and uncertainty and the existence of significant number of publications covering the theory of algebraic inequalities (Bechenbach and Bellman, 1961; Cloud et al, 1998; Engel, 1998; Hardy, 1999; Pashpatte, 2005; Steele, 2004; Kazarinoff, 1961). A comprehensive overview of the use of inequalities in mathematics has been presented in (Fink, 2000). 
Algebraic inequalities are capable of reducing aleatory and epistemic uncertainty and ranking systems, processes and decisions in terms of reliability and determining tight bounds of riskcritical parameters, in the absence of any knowledge related to the values of the controlling variables or their distributions. In addition, algebraic inequalities can be interpreted in a meaningful way and this interpretation can be linked to real systems and processes. A key step of this process is creating relevant meaning for the variables entering the algebraic inequality, followed by an interpretation of the left- and right-hand side of the inequality. This process effectively links an abstract algebraic inequality with a real physical system or process and not only opens opportunities for enhanced performance of systems and processes but also leads to the discovery of new fundamental properties of systems and processes and generation of new knowledge.

Consequently, this paper focuses on demonstrating the advantages of segmentation through algebraic inequalities and their meaningful interpretation in obtaining new knowledge, uncertainty reduction and enhancing the performance of systems and processes.

\section{Using segmentation through algebraic inequalities to achieve superior performance}

\subsection{Segmentation of a voltage source to maximise the power output}

Consider the abstract algebraic inequality

$$
\frac{a_{1}^{2}}{b_{1}}+\frac{a_{2}^{2}}{b_{2}}+\ldots+\frac{a_{n}^{2}}{b_{n}} \geq \frac{\left(a_{1}+a_{2}+\ldots+a_{n}\right)^{2}}{b_{1}+b_{2}+\ldots+b_{n}}
$$

valid for any sequence $a_{1}, a_{2}, \ldots, a_{n}$ of real numbers and any sequence $b_{1}, b_{2}, \ldots, b_{n}$ of positive real numbers. Inequality (1) is a transformation of the Cauchy-Schwarz inequality (Steele, 2004): 


$$
\left(x_{1} y_{1}+x_{2} y_{2}+\ldots+x_{n} y_{n}\right)^{2} \leq\left(x_{1}^{2}+x_{2}^{2}+\ldots+x_{n}^{2}\right)\left(y_{1}^{2}+y_{2}^{2}+\ldots+y_{n}^{2}\right)
$$

valid for any two sequences of real numbers $x_{1}, x_{2}, \ldots, x_{n}$ and $y_{1}, y_{2}, \ldots, y_{n}$. Indeed, the substitutions $x_{i}=\frac{a_{i}}{\sqrt{b_{i}}}(i=1, \ldots, n)$ and $y_{i}=\sqrt{b_{i}}(i=1, \ldots, n)$ in inequality (2) lead directly to inequality (1):

$\left(\frac{a_{1}}{\sqrt{b_{1}}} \sqrt{b_{1}}+\frac{a_{2}}{\sqrt{b_{2}}} \sqrt{b_{2}}+\ldots+\frac{a_{n}}{\sqrt{b_{n}}} \sqrt{b_{n}}\right)^{2} \leq\left(\left(a_{1} / \sqrt{b_{1}}\right)^{2}+\ldots+\left(a_{n} / \sqrt{b_{n}}\right)^{2}\right)\left(\left(\sqrt{b_{1}}\right)^{2}+\ldots+\left(\sqrt{b_{n}}\right)^{2}\right)$

This shows that inequality (1) is indeed a transformation of the Cauchy-Schwarz inequality Equality in (1) is attained only if $a_{1} / b_{1}=a_{2} / b_{2}=\ldots=a_{n} / b_{n}$.

A relevant meaning can now be created for the variables $a_{i}, b_{i}$ entering inequality (1). Inequality (1) creates the unique opportunity to segment a controlling factor $a=\sum_{i=1}^{n} a_{i}$ and a controlling factor $b=\sum_{i=1}^{n} b_{i}$, in order to achieve a larger effect (represented by the sum on the left hand side of Inequality (1).

Consider a case where factor $a$ is 'voltage' and factor $b$ is 'resistance'. Consider a source of voltage $V$ applied to $n$ elements in series, with resistances $r_{1}, r_{2}, \ldots, r_{n}$ (Figure 1a).

a)

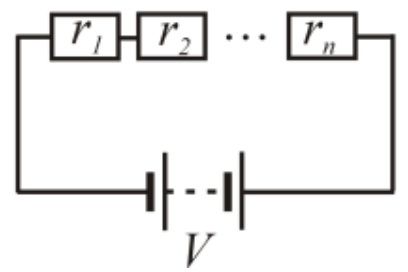

b)

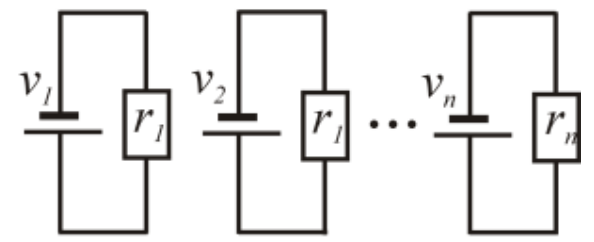

Figure 1. a) A single voltage source applied to elements connected in series; b) A voltage source $V$ segmented into $n$ smaller sources $v_{i}$ applied to the individual elements. 
Consider segmenting the source of voltage $V$ into $n$ smaller sources of voltage $v_{i}$ such that $V=v_{1}+\ldots+v_{n}$ (Figure $\left.1 \mathrm{~b}\right)$.

Let $a_{i}=v_{i}, i=1, \ldots, n$ be the voltages applied to $n$ separate elements with resistances $r_{i}$ (Figure 1b). If $b_{i}=r_{i}$, Inequality (1) can be rewritten as

$$
\frac{v_{1}^{2}}{r_{1}}+\frac{v_{2}^{2}}{r_{2}}+\ldots+\frac{v_{n}^{2}}{r_{n}} \geq \frac{V^{2}}{r_{1}+r_{2}+\ldots+r_{n}}
$$

Both sides of inequality (3) can be interpreted in the next theorem, relevant to electrical circuits:

Theorem 1. The power output from a source with voltage $V$, on elements connected in series, never exceeds the total power output from the sources $v_{i}\left(\sum_{i} v_{i}=V\right)$, resulting from segmenting the original source $V$ and applied to the individual elements.

This theorem holds irrespective of the individual resistances $r_{i}$ of the elements and the individual voltages $v_{i}$ into which the voltage $V$ is segmented. It is an example of generating new knowledge by a meaningful interpretation of an algebraic inequality.

Note that existence of asymmetry in the system is essential for increasing the power output through segmentation of the voltage source. No increase in the power output is present if $v_{1} / r_{1}=v_{2} / r_{2}=\ldots=v_{n} / r_{n}=i$. This means that rresistors with the same current $i$ do not yield an increase of the amount of power output.

To maximise the left-hand side of inequality (3), the squared voltages arranged in descending order $v_{1}^{2} \geq v_{2}^{2} \geq \ldots \geq v_{n}^{2}$ must correspond (be applied) to the resistances arranged in ascending order $\left(r_{1} \leq r_{2} \leq \ldots \leq r_{n}\right)$. For resistances arranged in ascending order, their reciprocals are arranged in descending order $\left(1 / r_{1} \geq 1 / r_{2} \geq \ldots \geq 1 / r_{n}\right)$ and according to the 
rearrangement inequality (Steele, 2004), the dot product $v_{1}^{2}\left(1 / r_{1}\right)+v_{2}^{2}\left(1 / r_{2}\right)+\ldots+v_{n}^{2}\left(1 / r_{n}\right)$ of two similarly ordered sequences is a maximum.

Thus, for resistances $r_{1}=10 \Omega, r_{2}=15 \Omega, r_{3}=25 \Omega$ and $r_{4}=50 \Omega$ and voltage source of $16 \mathrm{~V}$ segmented into $v_{1}=6 \mathrm{~V}, v_{2}=5 \mathrm{~V}, v_{3}=3 \mathrm{~V}$ and $v_{4}=2 \mathrm{~V}$, the maximum possible power is $P_{\max }=v_{1}^{2} / r_{1}+v_{2}^{2} / r_{2}+v_{3}^{2} / r_{3}+v_{4}^{2} / r_{4}=5.7 \mathrm{~W}$. Any other permutation of voltages to elements will result in smaller power.

If the source of $V=16 \mathrm{~V}$ is not segmented, applying the voltage to the four elements in series delivers power of $P=V^{2} /\left(r_{1}+r_{2}+r_{3}+r_{4}\right)=2.56 \mathrm{~W}$, which is less than half the maximum power of $P_{\max }=5.7 \mathrm{~W}$ delivered in the case of voltage source segmentation.

\subsection{Segmentation of a charge to maximise the energy stored in a capacitor}

In inequality (1), another relevant meaning can be created for the variables $a_{i}, b_{i}$. Factor $a$ could, for example, stand for 'electric charge' and factor $b$ could stand for 'capacitance'. In basic electronic (Floyd, 2004), a well known result is that the potential energy $U$ stored by a charge $Q$ in a capacitor with capacitance $C$ is given by $U=Q^{2} /(2 C)$.

Consider segmenting the charge $Q$ into $n$ smaller charges $q_{i}, i=1, \ldots, n$ such that $Q=q_{1}+\ldots+q_{n}$. In inequality (1), let $a_{i}=q_{i}, i=1, \ldots, n$ be the charges applied to $n$ separate capacitors with capacitances $C_{i}$. If $b_{i}=C_{i}$, inequality (1) can be rewritten as

$$
\frac{q_{1}^{2}}{2 C_{1}}+\frac{q_{2}^{2}}{2 C_{2}}+\ldots+\frac{q_{n}^{2}}{2 C_{n}} \geq \frac{Q^{2}}{2\left(C_{1}+C_{2}+\ldots+C_{n}\right)}
$$


Both sides of Inequality (4) can be interpreted in in the next new theorem, relevant to electrical capacitors:

Theorem 2. The energy stored by a charge $Q$ in a capacitor with capacitance $C$, never exceeds the total energy stored in multiple capacitors with the same equivalent capacity $\sum_{i} C_{i}=C \quad$ by segmenting the initial charge $Q$ over th individual capacitors.

This theorem holds irrespective of the individual capacitance $C_{i}$ of the capacitors and the charges $q_{i}$ into which the charge $Q$ is segmented.

Note that existence of asymmetry is essential for increasing the energy stored by segmenting the initial charge $Q$. No increase in the stored energy is present if $q_{1} / C_{1}=q_{2} / C_{2}=\ldots=q_{n} / C_{n}=U$. This means that capacitors loaded to the same potential difference $U=q_{i} / C_{i}$ on the plates, do not yield an increase of the amount of stored total energy.

To maximise the left hand side of inequality (4), the squared segmented charges arranged in descending order $q_{1}^{2} \geq q_{2}^{2} \geq \ldots \geq q_{n}^{2}$ must correspond (be applied) to the capacitances arranged in ascending order $\left(C_{1} \leq C_{2} \leq \ldots \leq C_{n}\right)$. For capacitances arranged in ascending order, the reciprocals $1 /\left(2 C_{1}\right) \geq 1 /\left(2 C_{2}\right) \geq \ldots \geq 1 /\left(2 C_{n}\right)$ are arranged in descending order and, according to the rearrangement inequality, the 'dot product' $q_{1}^{2} /\left(2 C_{1}\right)+q_{2}^{2} /\left(2 C_{2}\right)+\ldots+q_{n}^{2} /\left(2 C_{n}\right)$ of two similarly ordered sequences is a maximum.

Theorems 1 and 2 have never been stated in any book or publication in the mature fields of electrical engineering and electronics which demonstrates that the lack of knowledge of the domain-independent method of inequalities made these important properties invisible to domain experts. The theorems are examples of generating new knowledge by a meaningful interpretation of an algebraic inequality. 
The theorems have wide potential applications in the solar cell technology, to maximise the power output from photovoltaic cells (for example for low temperatue heating) and energy storage devices based on super-capacitors. The theorems also have potential applications in the electrical vehicles technology, to maximise the power output on demand.

\section{Segmentation and aggregation through super-additive and sub-additive inequalities}

In addition to inequality (1), there exist other algebraic inequalities yielding superior performance of a system or process by a segmentation of a controlling factor. Inequalities based on sub-additive and super-additive functions can be used for this purpose. Sub-additive and super-additive functions of two variables have, for example, been discussed in (Alsina and Nelsen, 2010). Despite this, to the best of our knowledge, sub-additive and super-additive functions have not yet been applied for optimizing processes by segmentation or aggregation of a controlling factor.

Consider a factor $x$ that has been segmented into a number of non-negative parts $x_{1}, x_{2}, \ldots, x_{n}\left(x=x_{1}+x_{2}+\ldots+x_{n}\right) ; n \geq 2$. The output associated with the factor $x$ and each of the parts $x_{i}$ is given by the function $f(\bullet)$. The output function $f(\bullet)$ is said to be sub-additive if for any set $x_{1}, x_{2}, \ldots, x_{n}$, the next inequality holds:

$$
f\left(x_{1}+x_{2}+\ldots+x_{n}\right) \leq f\left(x_{1}\right)+f\left(x_{2}\right)+\ldots+f\left(x_{n}\right)
$$

The output function $f(\bullet)$ is super-additive, if for any real numbers $x_{1}, x_{2}, \ldots, x_{n}$, the next inequality holds:

$$
f\left(x_{1}+x_{2}+\ldots+x_{n}\right) \geq f\left(x_{1}\right)+f\left(x_{2}\right)+\ldots+f\left(x_{n}\right)
$$

Note that if the output function $f(\bullet)$ is linear, equality is attained in inequalities (5) and (6). 
Multiplying the definition inequalities (5) and (6) by '-1', reverses the direction of the inequalities. Consequently, if $f(\bullet)$ is sub-additive, $-f(\bullet)$ is super-additive and if $f(\bullet)$ is super-additive, $-f(\bullet)$ is sub-additive. An example of a super-additive output function defined for $-\infty<x<\infty$ is the function $e^{x}$.

For the sake of clarity, before introducing some basic properties of sub-additive and superadditive inequalities, the well-known definitions of convex and concave functions will be recalled.

A function $f(x)$ with a domain $[a, b]$ is said to be convex (Figure $2 \mathrm{a}$ ), if for all values $x_{1}$ and $x_{2}$ in its domain $\left(x_{1}, x_{2} \in[a, b]\right)$, the next inequality holds:

$$
f\left(w x_{1}+(1-w) x_{2}\right) \leq w f\left(x_{1}\right)+(1-w) f\left(x_{2}\right), \text { where } 0 \leq w \leq 1 .
$$

(a)

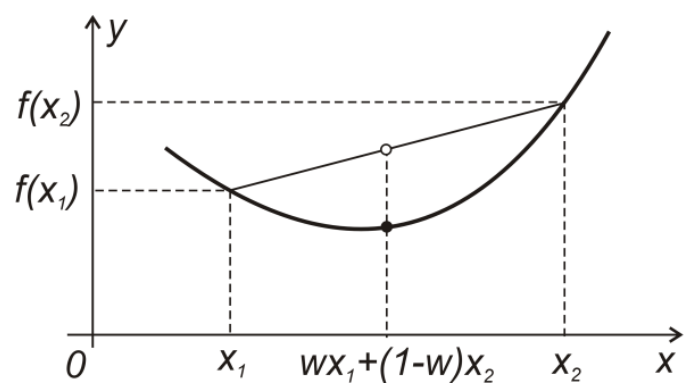

(b)

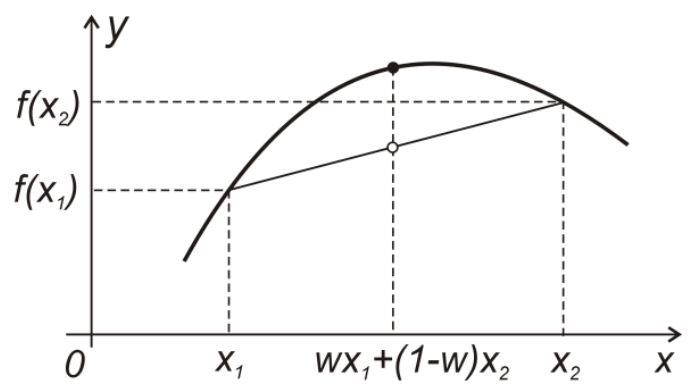

Figure 2. (a) A convex and (b) a concave function of a single argument.

If $f(x)$ is twice differentiable for all $x \in(a, b)$, and if the second derivative is non-negative $\left(f^{\prime \prime}(x) \geq 0\right)$, the function $f(x)$ is convex on $(a, b)$. For any convex function $f(x)$, the Jensen's inequality (Steele, 2004) states that

$$
f\left(w_{1} x_{1}+w_{2} x_{2}+\ldots+w_{n} x_{n}\right) \leq w_{1} f\left(x_{1}\right)+w_{2} f\left(x_{2}\right)+\ldots+w_{n} f\left(x_{n}\right),
$$

where $w_{i}(i=1, \ldots, n)$ are numbers (weights) that satisfy $0 \leq w_{i} \leq 1$ and $w_{1}+w_{2}+\ldots+w_{n}=1$. 
A function $f(x)$ with a domain $[a, b]$ is said to be concave (Figure $2 b$ ), if for all values $x_{1}$ and $x_{2}$ in its domain $\left(x_{1}, x_{2} \in[a, b]\right)$, the next inequality holds:

$$
f\left(w x_{1}+(1-w) x_{2}\right) \geq w f\left(x_{1}\right)+(1-w) f\left(x_{2}\right), \text { where } 0 \leq w \leq 1 .
$$

For any concave function $f(x)$, the Jensen's inequality states that

$$
f\left(w_{1} x_{1}+w_{2} x_{2}+\ldots+w_{n} x_{n}\right) \geq w_{1} f\left(x_{1}\right)+w_{2} f\left(x_{2}\right)+\ldots+w_{n} f\left(x_{n}\right)
$$

where $w_{i}(i=1, \ldots, n)$ are numbers (weights) that satisfy $0 \leq w_{i} \leq 1$ and $w_{1}+w_{2}+\ldots+w_{n}=1$.

If $f(x)$ is twice differentiable for all $x \in(a, b)$, and if the second derivative is not positive $\left(f^{\prime \prime}(x) \leq 0\right)$, the function $f(x)$ is concave on $(a, b)$.

A central property related to sub-additive and super-additive inequalities can now be stated.

If a function $f(\bullet)$, with a domain $[0, \infty)$ and range $[0, \infty)$ is concave, then the function is sub-additive $\left(f\left(x_{1}+x_{2}+\ldots+x_{n}\right) \leq f\left(x_{1}\right)+f\left(x_{2}\right)+\ldots+f\left(x_{n}\right)\right)$. If the function $f(\bullet)$ is convex, and if $f(0) \leq 0$ then the function is super-additive $\left.f\left(x_{1}+x_{2}+\ldots+x_{n}\right) \geq f\left(x_{1}\right)+f\left(x_{2}\right)+\ldots+f\left(x_{n}\right)\right)$.

\section{Proof.}

The correctness of the statement can be proved by an argument based on the fact that $f(\bullet)$ is a concave function. Note that $w_{1, k}=x_{k} / \sum_{i=1}^{n} x_{i}$ and $w_{2, k}=\left(\sum_{i=1}^{n} x_{i}-x_{k}\right) / \sum_{i=1}^{n} x_{i}$ can be treated as weights because $0 \leq w_{1, k} \leq 1,0 \leq w_{2, k} \leq 1$ and $w_{1, k}+w_{2, k}=1$. Because $f(\bullet)$ is a concave function, applying the classical Jensen's inequality for concave functions gives:

$$
f\left(x_{1}\right)=f\left(\frac{x_{1}}{\sum_{i=1}^{n} x_{i}} \times \sum_{i=1}^{n} x_{i}+\frac{\sum_{i=1}^{n} x_{i}-x_{1}}{\sum_{i=1}^{n} x_{i}} \times 0\right) \geq \frac{x_{1}}{\sum_{i=1}^{n} x_{i}} f\left(x_{1}+\ldots+x_{n}\right)+\frac{\sum_{i=1}^{n} x_{i}-x_{1}}{\sum_{i=1}^{n} x_{i}} f(0)
$$




$$
\begin{aligned}
& f\left(x_{2}\right)=f\left(\frac{x_{2}}{\sum_{i=1}^{n} x_{i}} \times \sum_{i=1}^{n} x_{i}+\frac{\sum_{i=1}^{n} x_{i}-x_{2}}{\sum_{i=1}^{n} x_{i}} \times 0\right) \geq \frac{x_{2}}{\sum_{i=1}^{n} x_{i}} f\left(x_{1}+\ldots+x_{n}\right)+\frac{\sum_{i=1}^{n} x_{i}-x_{2}}{\sum_{i=1}^{n} x_{i}} f(0) \\
& f\left(x_{n}\right)=f\left(\frac{x_{n}}{\sum_{i=1}^{n} x_{i}} \times \sum_{i=1}^{n} x_{i}+\frac{\sum_{i=1}^{n} x_{i}-x_{n}}{\sum_{i=1}^{n} x_{i}} \times 0\right) \geq \frac{x_{n}}{\sum_{i=1}^{n} x_{i}} f\left(x_{1}+\ldots+x_{n}\right)+\frac{\sum_{i=1}^{n} x_{i}-x_{n}}{\sum_{i=1}^{n} x_{i}} f(0)
\end{aligned}
$$

Adding all $n$ inequalities (7),(8),...,(9) yields

$$
f\left(x_{1}\right)+f\left(x_{2}\right)+\ldots+f\left(x_{n}\right) \geq f\left(x_{1}+x_{2}+\ldots+x_{n}\right)+(n-1) f(0)
$$

Since $f(0) \geq 0$ (the range of the function $f(\bullet)$ is $[0, \infty))$,

$$
f\left(x_{1}\right)+f\left(x_{2}\right)+\ldots+f\left(x_{n}\right) \geq f\left(x_{1}+x_{2}+\ldots+x_{n}\right)+(n-1) f(0) \geq f\left(x_{1}+x_{2}+\ldots+x_{n}\right)
$$

This completes the proof of inequality (5).

Note that if $f(0)>0$ equality in (10) cannot be attained:

$$
f\left(x_{1}\right)+f\left(x_{2}\right)+\ldots+f\left(x_{n}\right) \geq f\left(x_{1}+x_{2}+\ldots+x_{n}\right)+(n-1) f(0)>f\left(x_{1}+x_{2}+\ldots+x_{n}\right)
$$

In the same way, inequality (6) can also be proved if the function $f(x)$ is convex and $f(x) \leq 0$

The sub-additive inequality (5) and the super-additive inequality (6) have important potential applications. If the function $f(\bullet)$ measures the effect/output of a particular factor and $x_{i}$ denote the different magnitudes of the factor, inequalities (5) and (6) provide the unique opportunity to increase the effect of the factor by segmenting or aggregating it, depending on whether the function $f(\bullet)$ is concave or convex. If the function is concave, with a domain $[0, \infty)$ and range $[0, \infty)$, segmenting the factor results in a larger output. If the 
function is super-additive eonvex, with a domain $[0, \infty)$ and range $[0, \infty)$, aggregating the factor results in a larger output.

The knowledge about whether the function is sub-additive or super-additive eonvex or concave, combined with the requirement that the function has a domain $[0, \infty)$ and range $[0, \infty)$ is sufficient to reduce uncertainty and make a correct decision, irrespective of the magnitudes $x_{i}$ of the factor in the different segments.

The use of sub-additive and super-additive inequalities in process optimisation will be illustrated by using power-law dependences involving the controlled factor. Many real phenomena are approximated very well by power-law dependencies of the type

$$
y=a x^{p}
$$

where $a>0$ and $p>0$ are constants and $x$ is the controlling factor.

In what follows we show that depending on whether the power $p$ is greater or smaller than unity, sub-additive or super-additive inequalities can be used to perform segmentation or aggregation of the controlling factor in order to attain enhanced performance.

The quantity $y$ is a convex or concave function of the controlling factor $x(x \geq 0)$ depending on whether the second derivative $d^{2} y / d x^{2}=a p(p-1) x^{k-2}$ with respect to $x$ is positive or negative. This depends on whether the power $p$ is greater or smaller than 1 . If $p>1$, and $d^{2} y / d x^{2} \geq 0$, the power function $y$ is convex. If $0<p<1$ and $d^{2} y / d x^{2} \leq 0$, the power function $y$ is concave.

For a controlling factor $x$ varying in the interval $[0, \infty)$ the quantity $y$ is within the range $[0, \infty)$ and $f(0)=0$. In this case, the function (11) is convex, and, the following superadditive inequality holds:

$$
a\left(x_{1}+x_{2}+\ldots+x_{n}\right)^{p} \geq a x_{1}^{p}+a x_{2}^{p}+\ldots+a x_{n}^{p}
$$


This inequality means that segmenting the controlling factor $x$ into $n$ parts yields a smaller total effect.

If $0 \leq p<1$, the power function (11) is concave and the following sub-additive inequality holds:

$$
a\left(x_{1}+x_{2}+\ldots+x_{n}\right)^{p} \leq a x_{1}^{p}+a x_{2}^{p}+\ldots+a x_{n}^{p}
$$

which means that aggregating the controlling factor $x$ yields a smaller total effect.

Consider two illustrating examples. The first example involves the formation of undesirable brittle phase, during cooling of a work-piece with spherical shape and volume $V$ from a particular initial temperature. The quantity of undesirable brittle phase formed during cooling is given by the power function:

$$
z=a V^{p}
$$

where $a$ and $p(p>1)$ are constants which depend on the initial temperature, the nature of the material, and the shape of the work-piece. The controlling factor in the power-law dependence (14) is the volume $V$ of the work-piece.

The formation of brittle phase during cooling compromises the mechanical properties of the specimen because increased quantity of brittle phase translates into significantly reduced strength.

If, during processing, there is a choice between using a single work-piece of volume $V$ or several work-pieces whose combined volume is equal to $V$, the question of interest is which choice is associated with a smaller total quantity of brittle phase formation during cooling. The quantity $z$ of brittle phase is a convex function of the volume of the work-piece because the second derivative with respect to the volume $V$ is positive: $d^{2} z / d V^{2}=a p(p-1) V^{p-2}$. Because for a volume varying in the interval $[0, \infty)$ the quantity of brittle phase varies within 
the range $[0, \infty)$ and $z(0)=0$, the function (14) giving the quantity of brittle phase is superadditive. Consequently, the inequality

$$
a\left(V_{1}+V_{2}+\ldots+V_{n}\right)^{p} \geq a V_{1}^{p}+a V_{2}^{p}+\ldots+a V_{n}^{p}
$$

holds. The right side of inequality (15) can be interpreted as the sum of the quantities of brittle phase formed during cooling of $n$ work-pieces with volumes $V_{1}, V_{2}, \ldots, V_{n}$. The left side of inequality (15) can be interpreted as the quantity of brittle phase formed during cooling of a single work-piece with a similar shape and volume equal to the sum of the volumes $V_{1}, V_{2}, \ldots, V_{n}$ of the smaller work-pieces.

As a result, from inequality (15) it follows that a segmentation of a work-piece with volume $V$ into $n$ work-pieces with similar shape and volumes $V_{1}, V_{2}, \ldots, V_{n}, \quad$ ( $V=V_{1}+V_{2}+\ldots+V_{n}$ ) whose sum is equal to the volume of the original specimen, decreases the amount of unwanted brittle phase during cooling, irrespective of the individual volumes $V_{1}, V_{2}, \ldots, V_{n}$.

The next example is about using super-additive inequalities and is related to the drag force $F_{d}$ acting on a body with volume $V$ moving with constant velocity $v$ through fluid with density $\rho$. The drag force

$$
F_{d}=(1 / 2) C_{d} \rho v^{2} A
$$

is proportional to the drag coefficient $C_{d}$ (a dimensionless number which depends on the shape of the body), the density $\rho$ of the fluid, the square $v^{2}$ of the velocity and the crosssectional area $A$ of the body. Considering that for a body with volume $V$, the cross-sectional area is proportional to $V^{2 / 3}$, the drag force can be represented as a function of the volume $V$ of the body:

$$
F_{d}=a V^{2 / 3}
$$


where $p=2 / 3<1$ and $a=\left(3^{2 / 3} / 2\right) \times(4 \pi)^{1 / 3} C_{d} \rho v^{2}$.

The drag force given by equation (17) is a concave function because the second derivative with respect to the volume $V$ is negative: $d^{2} F_{d} / d V^{2}=-2 a /\left(9 V^{4 / 3}\right)$. In addition, for a volume varying in the interval $[0, \infty)$ the drag force varies in the range $[0, \infty)$ and $F_{d}(0)=0$. Consequently, the drag force function (17) is sub-additive and the inequality

$$
a\left(V_{1}+V_{2}+\ldots+V_{n}\right)^{2 / 3} \leq a V_{1}^{2 / 3}+a V_{2}^{2 / 3}+\ldots+a V_{n}^{2 / 3}
$$

holds. The right side of inequality (18) can be interpreted as the total drag force acting on $n$ bodies with volumes $V_{1}, V_{2}, \ldots, V_{n}$. The left side of inequality (18) can be interpreted as the drag force acting on a body with a similar shape and volume equal to the sum of the volumes $V_{1}, V_{2}, \ldots, V_{n}$ of the separate bodies.

As a result, from inequality (18) it follows that aggregating $n$ bodies with a similar shape and volumes $V_{1}, V_{2}, \ldots, V_{n}$ into a single large body with a similar shape and larger volume $V=V_{1}+V_{2}+\ldots+V_{n}$, decreases the total drag force, irrespective of the actual magnitudes of the volumes $V_{1}, V_{2}, \ldots, V_{n}$.

Inequalities (15) and (18) are examples of optimising processes by a meaningful interpretation of algebraic inequalities.

\section{Conclusions}

1. The paper demonstrates for the first time segmentation achieving uncertainty reduction and superior performance through segmentation based on algebraic inequalities.

2. Meaningful interpretation of algebraic inequalities can be used for generating new knowledge in unrelated application domains. 
3. The meaningful interpretation of a version of the Cauchy-Schwarz inequality led to the discovery of new properties of electrical circuits. The power output from a single voltage source, on elements connected in series, is smaller than the total power output from the segmented source applied to the separate elements.

4. Existence of asymmetry is essential for increasing performance through segmentation by using the Cauchy-Schwarz inequality. Loaded elements experiencing the same current do not yield an increase of the power output from the segmented sources.

5. Segmentation through a transformed version of the Cauchy-Schwarz inequality led to a new theorem related to electrical capacitors. The energy stored by a charge of given size on a single capacitor is smaller than the total energy stored in multiple capacitors with the same equivalent capacity, by segmenting the initial charge over the separate capacitors.

6. By a meaningful interpretation of sub-additive and super-additive inequalities, superior performance and important properties have been established for processes described by a power-law dependence.

7. Important properties have been established for a process described by the power law $y=a x^{p}$ by using sub-additive and super-additive inequalities. If $p>1$, the segmentation of the controlling factor $x$ leads to a smaller total yield from the process. For $0 \leq p<1$ aggregation of the controlling factor $x$ leads to a smaller total yield from the process. 


\section{References}

Alsina C. and Nelsen R.B. (2010). Charming Proofs: A Journey into Elegant Mathematics, Washington, DC: The Mathematical Association of America.

Bechenbach E. and R.Bellman. (1961) An introduction to inequalities. New York: The L.W.Singer company.

Budynas R.G. (1999) Advanced strength and applied stress analysis, 2nd ed. New York: McGraw-Hill.

Budynas R.G. and Nisbett J.K. (2015) Shigley's Mechanical engineering design, 10th ed. New York: McGraw-Hill.

Childs P.R.N. (2014) Mechanical design engineering handbook. Amsterdam: Elsevier.

Cloud M., Byron C. and Lebedev L.P. (1998) Inequalities: with applications to engineering. New York: Springer-Verlag.

Collins J.A. (2003) Mechanical design of machine elements and machines. New York: John Wiley \& Sons, Inc.

Dhillon B.S. (2017) Engineering systems reliability, safety, and maintenance. New York: CRC Press.

Ebeling C.E. (1997) Reliability and maintainability engineering. Boston: McGraw-Hill.

Engel A. (1998) Problem-solving strategies. New York: Springer.

Floyd T. (2004) Electronics Fundamentals: Circuits, Devices and Applications. 6th ed., New Jersey: Pearson Education, Inc.

Fink A.M., (2000) An essay on the history of inequalities, Journal of Mathematical Analysis and Applications, Vol.249, pp.118-134.

French M. (1999) Conceptual design for engineers, 3rd ed. London: Springer-Verlag London Ltd.

Gullo L.G. and Dixon J. (2018) Design for safety. Chichester: Wiley.

Hardy, G., Littlewood J.E. and Pólya G. (1999) Inequalities. New York: Cambridge University Press.

Kazarinoff N.D. (1961) Analytic Inequalities. New York: Dover Publications, Inc.

Lewis E.E. (1996) Introduction to Reliability Engineering. New York:Wiley.

Modarres M., Kaminskiy M.P. and Krivtsov V. (2017) Reliability engineering and risk analysis, a practical guide, 3rd ed. CRC Press.

Mott R.L, Vavrek E.M. and Wang J. (2018) Machine Elements in Mechanical Design, 6th ed. Pearson Education Inc. 
Norton R.L. Machine design, an integrated approach, 3rd ed. Pearson International edition, 2006.

O’Connor P.D.T. (2002) Practical Reliability Engineering, 4ed. New York: Wiley.

Pahl G., Beitz W., Feldhusen J. and Grote K.H. (2007) Engineering design. Berlin: Springer.

Pachpatte B.G. (2005) Mathematical inequalities. North Holland Mathematical Library, vol.67, Amsterdam: Elsevier.

Samuel A. and Weir J. (1999) Introduction to engineering design: Modelling, synthesis and problem solving strategies. London: Elsevier.

Steele J.M. (2004) The Cauchy-Schwarz master class: An introduction to the art of mathematical inequalities. New York: Cambridge University Press.

Thompson G., (1999) Improving maintainability and reliability through design. London: Professional Engineering Publishing Ltd.

Todinov M.T. (2019a) Methods for reliability improvement and risk reduction. Wiley.

Todinov M.T. (2019b). Domain-independent approach to risk reduction, Journal of Risk Research. doi:10.1080/13669877.2019.1628093. 